\title{
Application of Glycemic Variability Percentage: Implications for Continuous Glucose Monitor Utilization and Analysis of Artificial Pancreas Data
}

\author{
Arturo Garcia, BS, Andrew K. Balo, BS, Bruce A. Buckingham, MD, \\ Irl B. Hirsch, MD, and Thomas A. Peyser, $\mathrm{PhD}^{4}$
}

\begin{abstract}
Background: The problem of glycemic variability has been widely acknowledged in patients with diabetes with severe insulin deficiency. In a companion article, we proposed a novel metric, the glycemic variability percentage (GVP), for assessing glycemic variability that accounts for both the amplitude and frequency of glycemic fluctuations. Method: We applied the new metric, the GVP, to a previously reported case of a subject using an earlier generation continuous glucose monitoring (CGM) device, in which successive periods of use were associated with an apparent decrease in glycemic variability. Results were compared with histogram distributions for the rate of change of glucose as well. The GVP was also applied to data from a published study of a bihormonal artificial pancreas system comparing results from open loop and closed loop in adolescents and in adults.

Results: The GVP was able to quantify the changes in glycemic variability during successive periods of CGM use. Application of the GVP to a published study of a bihormonal artificial pancreas found an increase in glycemic variability compared with other accepted metrics which suggested a decrease in glycemic variability. Conclusion: The GVP may be a clinically useful tool in characterizing the change in glycemic variability in subjects using CGM devices. Compared with metrics, such as the standard deviation, that focus solely on the amplitude of oscillations, the GVP, which measures both frequency and amplitude, may also be a more useful tool in assessing the true level of glycemic variability in artificial pancreas studies.
\end{abstract}

Keywords: Glycemic variability, Continuous glucose monitoring, Artificial pancreas.

\section{Introduction}

A $S$ NOTED IN the companion article published in this issue, there are numerous metrics for glycemic variability. ${ }^{1}$ As shown by Peyser et al., different metrics for glycemic variability may weigh more heavily or even measure exclusively the amplitude of glycemic fluctuations and weigh less heavily or even totally exclude the frequency of oscillations. The proposed new metric, the glycemic variability percentage (GVP), gives equal weight to both the amplitude and frequency of glycemic fluctuations. As a result, the new GVP metric may be able to differentiate between cases that are otherwise not distinguishable using several of the commonly accepted metrics for glycemic variability.

The GVP metric is similar mathematically to the distancetraveled approach proposed by Marling et al. ${ }^{2}$ and the mean amplitude glucose (MAG) change proposed by Hermanides et al. and DeVries. ${ }^{3,4}$ The GVP metric differs from the distance-traveled approach and the MAG change metric in that the results are expressed as a percentage compared with the minimum line length for a given duration. This may make the GVP metric easier to understand conceptually and clinically than distance traveled or MAG.

\footnotetext{
${ }^{1}$ Dexcom, Inc., San Diego, California.

${ }^{2}$ Department of Pediatric Endocrinology, Stanford University, Stanford, California.

${ }^{3}$ Department of Medicine, Division of Metabolism, Endocrinology and Nutrition, University of Washington, Seattle, Washington.

${ }^{4}$ Consultant, Menlo Park, California.
}

(C) Arturo Garcia, et al., 2018; Published by Mary Ann Liebert, Inc. This Open Access article is distributed under the terms of the Creative Commons License (http://creativecommons.org/licenses/by/4.0), which permits unrestricted use, distribution, and reproduction in any medium, provided the original work is properly credited. 
The GVP is based on the fact that the length of the continuous glucose monitor temporal trace over a given interval of time depends on the degree of glycemic variability. The GVP is given by the length of the line $L$ represented by the continuous glucose monitoring trace normalized to the ideal line length $L_{0}$ with zero glycemic variability. The GVP is then expressed as a percentage of variability above the zero glycemic variability ideal straight line for that duration. Since temporal traces with high glycemic variability have greater lengths than traces with low glycemic variability, higher values of GVP are indicative of higher levels of variability.

The GVP metric may be a useful tool for assessing glycemic variability in subjects already using continuous glucose monitoring devices or just beginning the use of the technology. The GVP metric may also be useful as another tool in assessing the performance of emerging commercial artificial pancreas technologies using unihormonal or bihormonal approaches as well as different algorithm control strategies. ${ }^{5}$ Finally, the GVP may be a better metric to assess clinical outcomes such as hypoglycemia risk and even markers of oxidative stress and inflammation that fuel the pathogenesis of the vascular complications of diabetes.

\section{Methods}

The new metric, the GVP, was applied to a previously reported case of a subject using an earlier generation continuous glucose monitoring (CGM) device, in which successive periods of use were associated with an apparent decrease in glycemic variability. ${ }^{6}$ In addition, changes in glycemic variability as measured by GVP were compared with changes in the glucose rate of change histogram based on CGM data. Finally, the GVP was also applied to data from a published study of a bihormonal artificial pancreas system comparing results from open loop and closed loop in adolescents and in adults. ${ }^{7}$ Results from the GVP metric were compared with published results using the standard deviation (SD) as the metric for glycemic variability.

\section{Results}

Ten years ago, in one of the earliest articles to show the clinical benefit of CGM devices, Garg et al. used the first-generation Dexcom continuous glucose monitoring system, the Dexcom
STS, and found decreasing levels of glycemic variability in sequential use of the 3-day sensor. ${ }^{6}$ Subjects used the Dexcom STS for the first 3 days in blinded mode. In two subsequent 3-day use periods of the sensor, subjects were shown the data and allowed to respond to the CGM values. In a subset of subjects, they observed a decrease in glycemic variability for both successive uses of the unblinded system. In their 2006 article, Garg et al. included a figure showing a dramatic reduction in glucose variability in one subject between the first 3 days of blinded use and the next 5 days of unblinded use with data displayed to the patient. ${ }^{6}$ During the first 3 days of sensor wear when the data were blinded, the subject exhibited a high level of glycemic variability as indicated by the GVP value of $59 \%$. During the next 3 days of sensor wear, the data were available to the subject, and there was a modest but discernible decrease in glycemic variability as measured by GVP to $45 \%$. However, during the first 2 days of the final sensor wear, there was a notable decrease in glycemic variability as measured by GVP to $29 \%$.

The data in Figure 1 above illustrate another interesting aspect of glycemic variability, namely, the relationship between high levels of glycemic variability and high rates of change that has been previously noted by other investigators. ${ }^{8}$ As noted by Peyser et al. in the companion article published in this journal, the mathematical determination of the line length using a Pythagorean decomposition of each 5-min line element means that the each line element is an approximation of the instantaneous rate of change of glucose over the measurement interval between two successive CGM values. Thus, this method is closely related to the proposals by Kovatchev et al. and Whitelaw et al. to use the rate of change of glucose to evaluate glycemic variability. 8,9

The reduced glycemic variability seen in the progression of traces in Figure 1 is also associated with a significant reduction in the occurrence of high rates of change, as shown in the histograms. Figure 2 below represents the three histogram distributions of the rates of change associated with the temporal traces in Figure 1.

During 3 days of blinded use of CGM, only $57.5 \%$ were within the stable region from -1 to $+1 \mathrm{mg} /(\mathrm{dL} \cdot \mathrm{min}) .84 .3 \%$ were within -2 to $+2 \mathrm{mg} /(\mathrm{dL} \cdot \mathrm{min})$, and $15.7 \%$ exhibited absolute rates of change greater than $2 \mathrm{mg} /(\mathrm{dL} \cdot \mathrm{min})$. During the first 3 days of unblinded use of CGM, there was a slight increase in the percentage within the stable region of -1 to

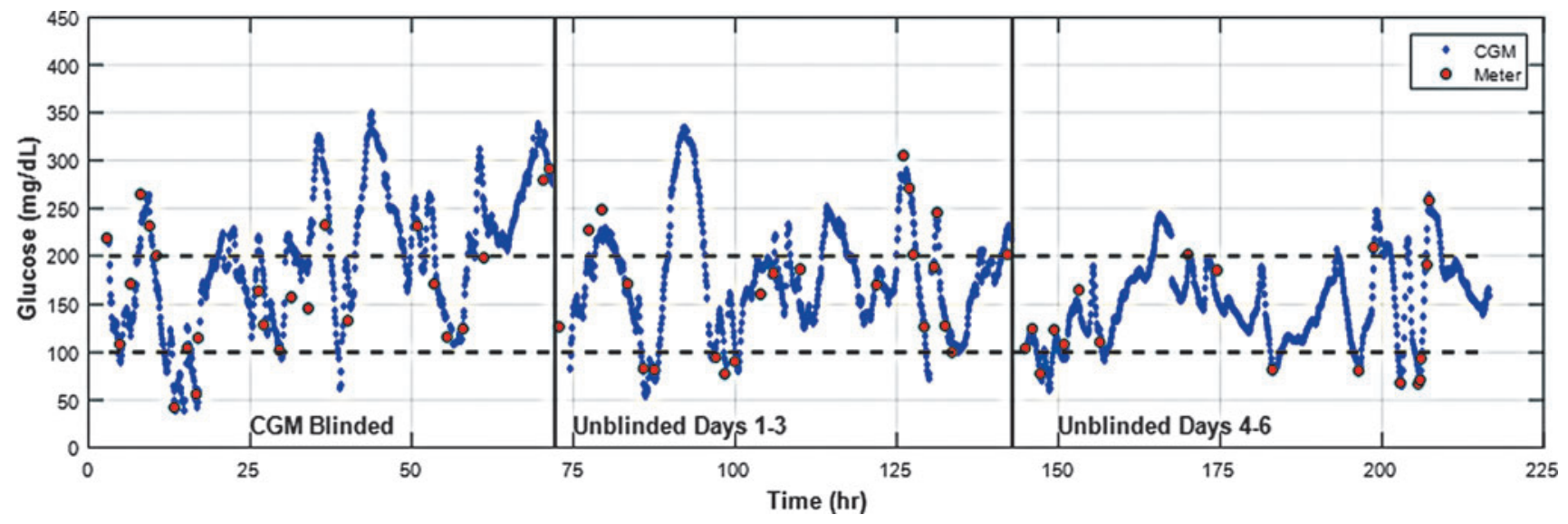

FIG. 1. Evidence of reduction in glycemic variability associated with continuous glucose monitoring use for 5 days following initial 3 day blinded use from Garg et al. ${ }^{6}$ The glycemic variability as measured by glycemic variability percentage (GVP) decreased from high glycemic variability GVP $=59 \%$ in the blinded phase to moderate glycemic variability $\mathrm{GVP}=45 \%$ in the first unblinded phase to low glycemic variability $\mathrm{GVP}=29 \%$ in the second unblinded phase. 

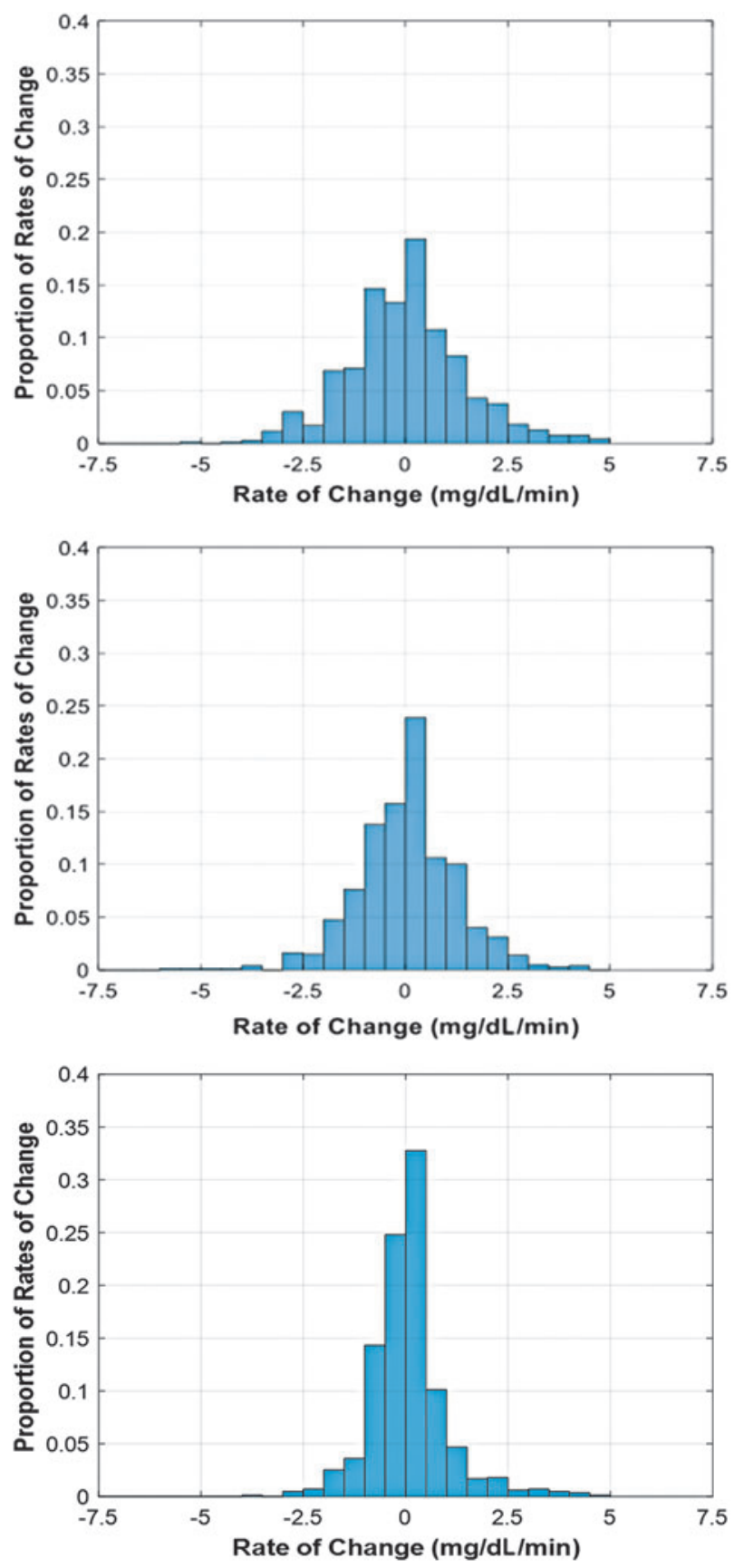

FIG. 2. (Top) Rate of change of glucose corresponding to initial 3 days blinded CGM use from data shown in Figure 1. (Middle) Rate of change of glucose corresponding to initial 3 days of unblinded CGM use from data shown in Figure 1. (Bottom) Rate of change of glucose corresponding to the final 2 days of unblinded CGM use from data shown in Figure 1. CGM, continuous glucose monitoring.

$+1 \mathrm{mg} /(\mathrm{dL} \cdot \min )$ to $63.8 \%, 90.9 \%$ were within -2 to $+2 \mathrm{mg} /$ (dL $\cdot \min$ ), and only $9.1 \%$ exhibited absolute rates of change greater than $2 \mathrm{mg} /(\mathrm{dL} \cdot \mathrm{min})$. Finally, during the last 2 days of unblinded use, there was a significant increase in the percentage within the stable region of -1 to $+1 \mathrm{mg} /(\mathrm{dL} \cdot \mathrm{min})$ to $81.0 \%, 94.0 \%$ were within -2 to $+2 \mathrm{mg} /(\mathrm{dL} \cdot \mathrm{min})$, and only $6.0 \%$ exhibited absolute rates of change greater than $2 \mathrm{mg} /$ $(\mathrm{dL} \cdot \mathrm{min})$. Continued CGM usage was associated not only with reduced glycemic variability but also with a redistribution of rates of change of glucose from high rates to lower rates.

The GVP was also applied to open-loop and closed-loop control data from a published study on the bionic pancreasa bihormonal artificial pancreas system. ${ }^{7}$ Figure 3 below shows the temporal trace for one subject in the study on open loop (top) and on closed loop (bottom). The mean glucose for the subject in the open-loop arm was $192 \mathrm{mg} / \mathrm{dL}$ compared with $155 \mathrm{mg} / \mathrm{dL}$ for the subject in the bionic pancreas arm. Two of the measures of glycemic variability, Mean Amplitude of Glycemic Excursions (MAGE) and SD, found a decrease in glycemic variability for the open-loop subject in Figure 3 compared with the bionic pancreas subject. The value of MAGE and SD appeared to decrease from 213 and 88 , in the open-loop example, respectively, to 186 and 70 in the bionic pancreas example. However, the coefficient of variation $(\mathrm{CV})$ metric for glycemic variability was essentially unchanged, that is, 0.46 in the open loop and 0.45 in the closed loop. The GVP metric for mean glycemic variability showed an apparent increase in glycemic variability from $44 \%$ for the open-loop arm to $53 \%$ for the closed loop or bionic pancreas arm.

Figure 4 gives the cumulative distribution of mean glucose and glycemic variability as measured by the GVP for all subjects in the open loop, the first 3 days of closed-loop control and the final 2 days of closed-loop control. As discussed by Russell et al., subjects in the closed loop or bionic pancreas arm had a markedly lower mean glucose than subjects in the open-loop arm. In the open-loop arm, the mean glucose was $160.9( \pm 25.3) \mathrm{mg} / \mathrm{dL}$, and the average glycemic variability measured by GVP was $46 \%( \pm 11 \%)$. The bionic pancreas algorithm is adaptive and requires at least 1 day of adaptation to the individual subject, therefore changes in both the mean glucose and glycemic variability might be expected after several days of use. During the first 3 days of closed loop control, the mean glucose was $145.8( \pm 18.5) \mathrm{mg} / \mathrm{dL}$ and decreased further during the fourth and fifth day to 138.6 $( \pm 13.8) \mathrm{mg} / \mathrm{dL}$.

Analysis of the data remained statistically unchanged with the glycemic variability between the first 3 days of use with GVP equal to $53 \%( \pm 18 \%)$ and the fourth and fifth day of use with GVP equal to $58 \%( \pm 21 \%)$. However, the mean GVP in the bionic pancreas cohort over all 5 days was $55 \%( \pm 17 \%)$ compared with $46 \%( \pm 11 \%)$ in the open-loop cohort. In addition, there were more subjects in the bionic pancreas or closed-loop arm with a higher level of glycemic variability than those in the open-loop arm. In fact, $63.5 \%$ of subjects in the closed-loop cohort exhibited glycemic variability in the highest quartile compared with only $26.9 \%$ in the open-loop cohort. The greater proportion of subjects with high levels of glycemic variability in the bionic pancreas cohort than in the open-loop cohort can be seen clearly in the cumulative distribution plot in Figure 4. The increase in glycemic variability in the closed-loop arm as measured by GVP may be related to the higher frequency of glucose oscillations, resulting from the competing action of high levels of insulin administered by the algorithm to rapidly decrease blood glucose and the frequent administration of glucagon by the algorithm to prevent the occurrence of hypoglycemia.

The increase in glycemic variability in closed loop compared with open loop as measured by the glucose variability percentage metric is also apparent from interquartile 

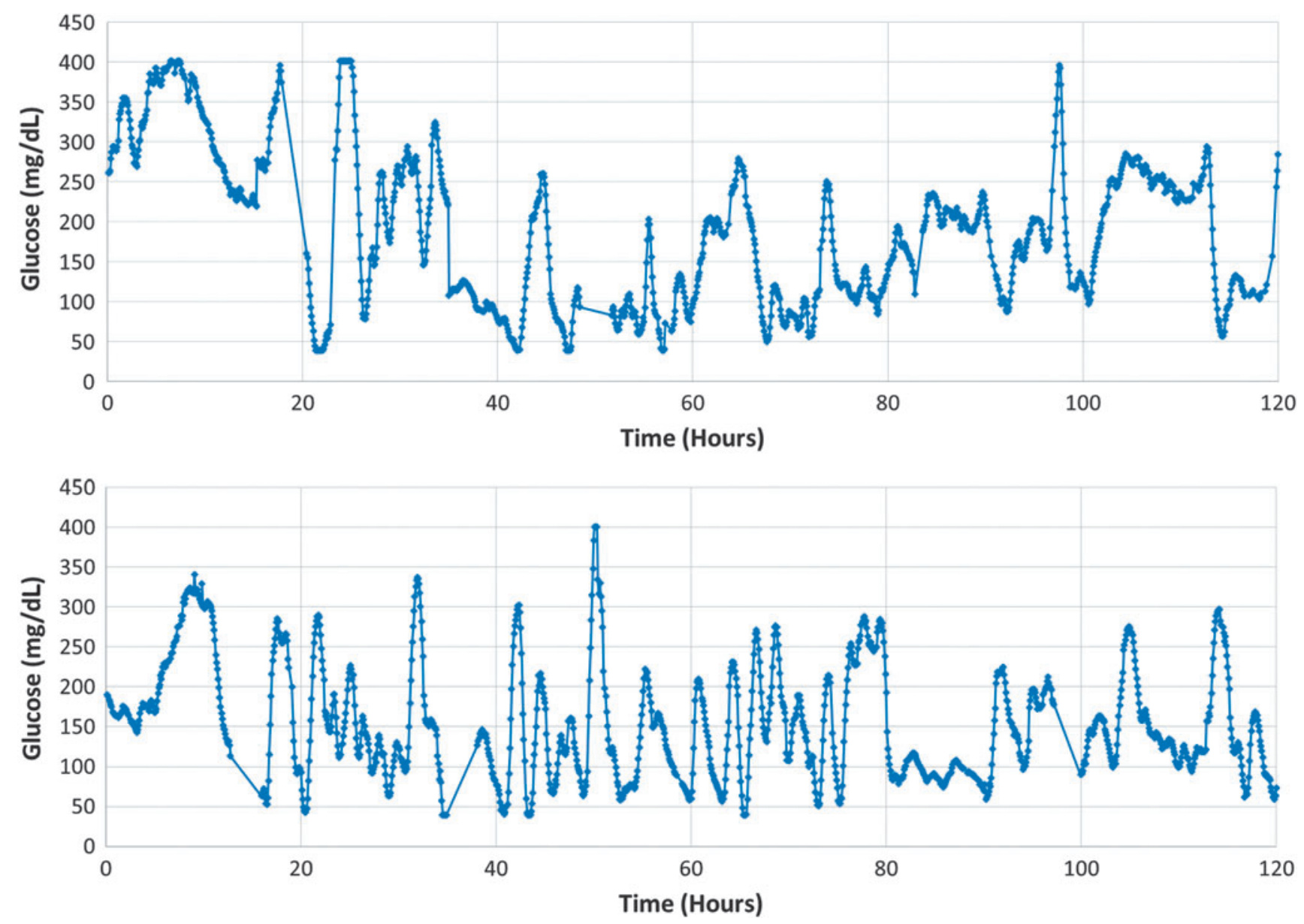

FIG. 3. Temporal traces from the same subject on open-loop (top) and closed-loop (bottom) control from a recently published study on the bionic pancreas. In the example shown in this article, the mean glucose decreased from $192 \mathrm{mg} / \mathrm{dL}$ in the open-loop control to $155 \mathrm{mg} / \mathrm{dL}$ during closed-loop control. The glycemic variability remained high and increased from GVP of $44 \%$ during open loop to $53 \%$ during closed loop.

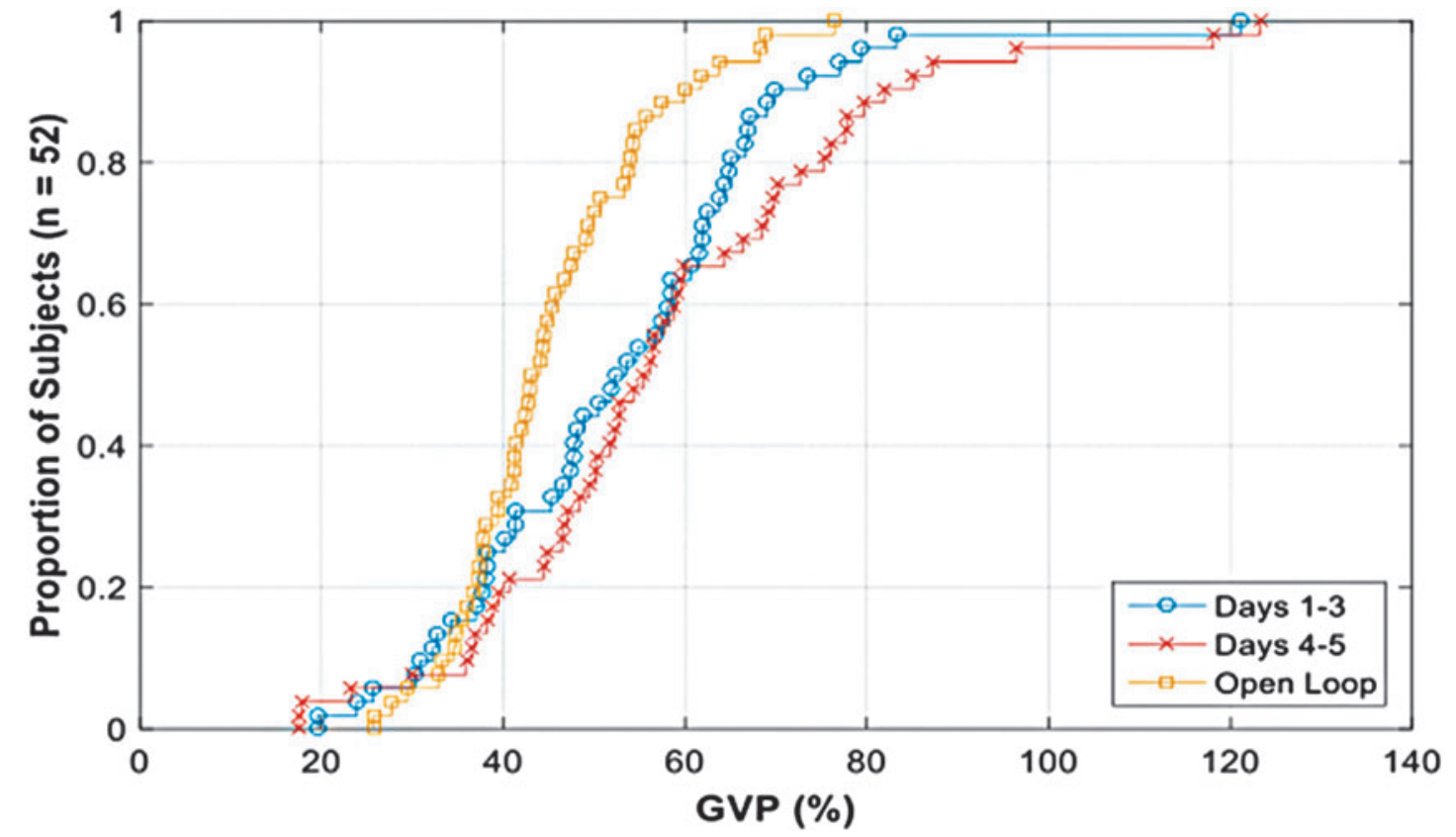

FIG. 4. Cumulative distributions for the GVP for open-loop arm, for the first 3 days of the closed-loop arm and the last 2 days of the closed control arm of the bionic pancreas study reported by Russell et al. ${ }^{7}$ 
Table 1. Interquartile Analysis of the Glycemic Variability Percentage in Four Study Subject Cohorts: Adults with Diabetes from Dexcom CGM Studies, Adolescents with Diabetes from Dexcom CGM Studies, ${ }^{1}$ Subjects with Diabetes in the Open-Loop Arm of the Bionic Pancreas Study, and Subjects with Diabetes in the Closed-Loop Study of the Bionic Pancreas Study ${ }^{7}$

\begin{tabular}{lcccc}
\hline $\begin{array}{l}\text { Interquartile } \\
\text { range }\end{array}$ & $\begin{array}{c}\text { Adults with type 1 } \\
\text { diabetes }(G V P), \%\end{array}$ & $\begin{array}{c}\text { Adolescents with type } 1 \\
\text { diabetes }(G V P), \%\end{array}$ & $\begin{array}{c}\text { Subjects in OL bionic } \\
\text { pancreas }(G V P), \%\end{array}$ & $\begin{array}{c}\text { Subjects in CL bionic } \\
\text { pancreas }(G V P), \%\end{array}$ \\
\hline 25th Pct. & 35 & 42 & 38 & 44 \\
50th Pct. & 43 & 52 & 44 & 53 \\
75th Pct. & 49 & 63 & 51 & 66 \\
100th Pct. & 70 & 112 & 76 & 99
\end{tabular}

CGM, continuous glucose monitoring; CL, Closed Loop; GVP, glycemic variability percentage; OL, Open Loop; Pct, percentile.

analysis. Table 1 contains comparative glycemic variability data using the GVP metric for adult and adolescent subjects in independent open-loop CGM studies, subjects in the open-loop arm of the bionic pancreas study and subjects in the closed-loop arm of the bionic pancreas study.

In the bionic pancreas trial, subjects in the open-loop arm had a mean (50th percentile) GVP of $44 \%$. The mean glycemic variability in the open-loop arm is consistent with findings from independent open-loop studies with CGM devices in both adults and adolescents. However, the mean (50th percentile) GVP in the closed-loop arm of the bionic pancreas study was $53 \%$. This is comparable to the mean glycemic variability seen in adolescents in open-loop CGM studies. In addition, the GVP in the 75th and 100th percentile of the data sets was $66 \%$ and $99 \%$, respectively. This is significant because this metric shows an apparent increase in the highest level of glycemic variability in the closed-loop arm compared with the open-loop arm and with comparable data from CGM studies of subjects also on open-loop therapy.

The increase in the GVP metric in closed-loop arm of the bionic pancreas study can be modeled heuristically with a simple square-wave profile of various amplitudes, mean glucose, and numbers of periods of oscillations per day. The top graph in Figure 5 gives the square-wave profile with a $65 \mathrm{mg} / \mathrm{dL}$ amplitude, $135 \mathrm{mg} / \mathrm{dL}$ mean glucose, and three periods of 48-h duration per day corresponding hypothetically to the open arm of the study. The middle graph in Figure 5 gives the square-wave profile with a $45 \mathrm{mg} / \mathrm{dL}$ amplitude, $115 \mathrm{mg} / \mathrm{dL}$ mean glucose, and six periods of 4-h duration per day corresponding to three meals per day plus an additional oscillatory cycle associated with the glucagon response following each meal. The bottom graph in Figure 5 gives the square-wave profile for three meals per day with a $40 \mathrm{mg} / \mathrm{dL}$ amplitude, $140 \mathrm{mg} / \mathrm{dL}$ mean glucose, and three periods of oscillations corresponding hypothetically to the case of a patient with type 1 diabetes and low glycemic variability.

The 8-h period square-wave example in the top figure has a GVP of $51 \%$ comparable to the open-loop results from the clinical study. The 4-h period square-wave example in the middle figure has a GVP of $68.5 \%$, illustrative qualitatively of the measured GVP for the closed-loop results from the study. These results are comparable to the actual measured GVP of $44 \%$ for the open-loop arm and 53\% for the closed loop or bionic pancreas arm. Finally, the square-wave profile shown in the bottom figure has a GVP value of $30.5 \%$ consistent with the results of the interquartile analysis in the companion article for type 1 diabetes subjects in the lowest quartile of glycemic variability.
Figure 6 shows the difference in GVP using a box-whisker plot between subjects during the open-loop phase and during the closed-loop phase. There is a noticeable increase in the range of glycemic variability as measured by GVP in the closed-loop arm compared with the open-loop arm. There is a clear increase in the $25 \mathrm{th}, 50 \mathrm{th}$, and 75 th percentile analysis of the GVP as well as in the maximum values.

It is interesting to note that the standard metrics for assessing glycemic variability do not capture this effect. On the contrary, the standard metrics such as MAGE, CV, and SD show a decrease in glycemic variability between the openloop and closed-loop arms of the bionic pancreas study. The standard metrics, especially MAGE and SD, can be regarded as measures of relative glycemic variability giving greater weight to the amplitude of oscillations, but not the frequency of oscillations. CV can be regarded as a measure of relative glycemic variability giving greater weight to the frequency of oscillations as opposed to the amplitude. Table 2 below shows the mean glycemic variability for all four metrics (MAGE, CV, SD, and GVP). MAGE, SD, and CV show a decrease in variability between the open loop and bionic pancreas arms of the study. The GVP metric balances both the amplitude and frequency of oscillations and shows an increase in aggregate glycemic variability in the closed-loop arm compared with the open-loop arm. By contrast, the GVP metric shows a significant increase in glycemic variability. The GVP metric is the only one of the four that captures the effect of both the frequency and amplitude of oscillations on the overall assessment of glycemic variability.

\section{Discussion}

Successful use of a continuous glucose monitoring system should result not only in reduced mean glucose values but also in reduced glycemic variability. A recently published study by El-Laboudi et al. calculated the change in glycemic variability using a range of available metrics in subjects in the JDRF CGM study and found decreases virtually in all measures except the MAG change metric. ${ }^{10}$ They speculated that "the use of real-time glucose trends and alarms addresses glucose dispersion and risk while the underlying temporal glucose variability remains unchanged" which would explain the reduction in measures of glucose variability relying on the amplitude of glucose fluctuations, but not the frequency.

As Kovatchev and Cobelli have recently pointed out, the temporal component of glucose variability is important, since the risk of hypoglycemia depends strongly on both the mean 

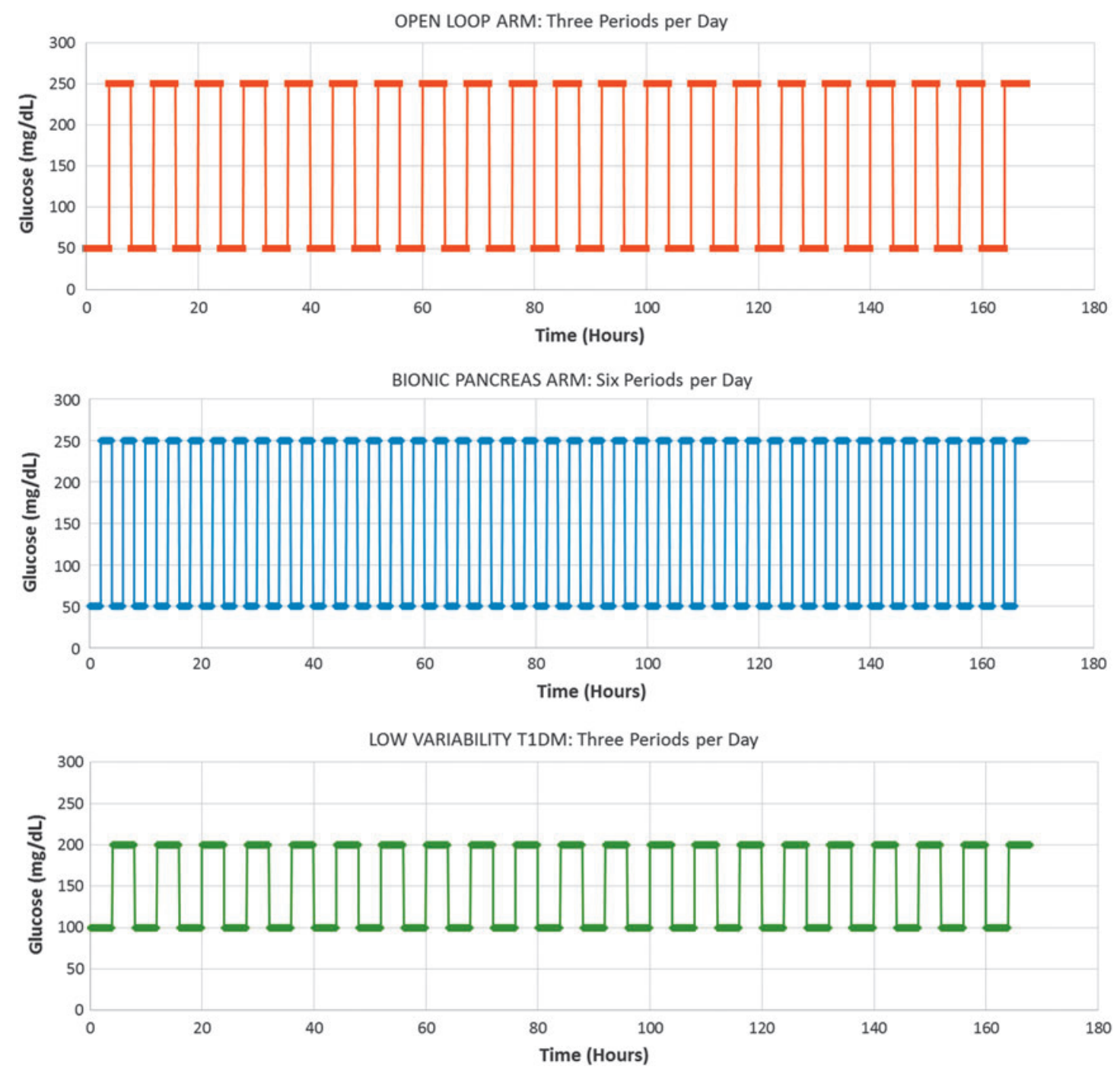

FIG. 5. Three square-wave examples to illustrate qualitatively the glycemic variability associated with the open-loop arm of the Russell et al. study, the closed-loop arm of the same study, and hypothetical subject with type 1 diabetes and low glycemic variability.

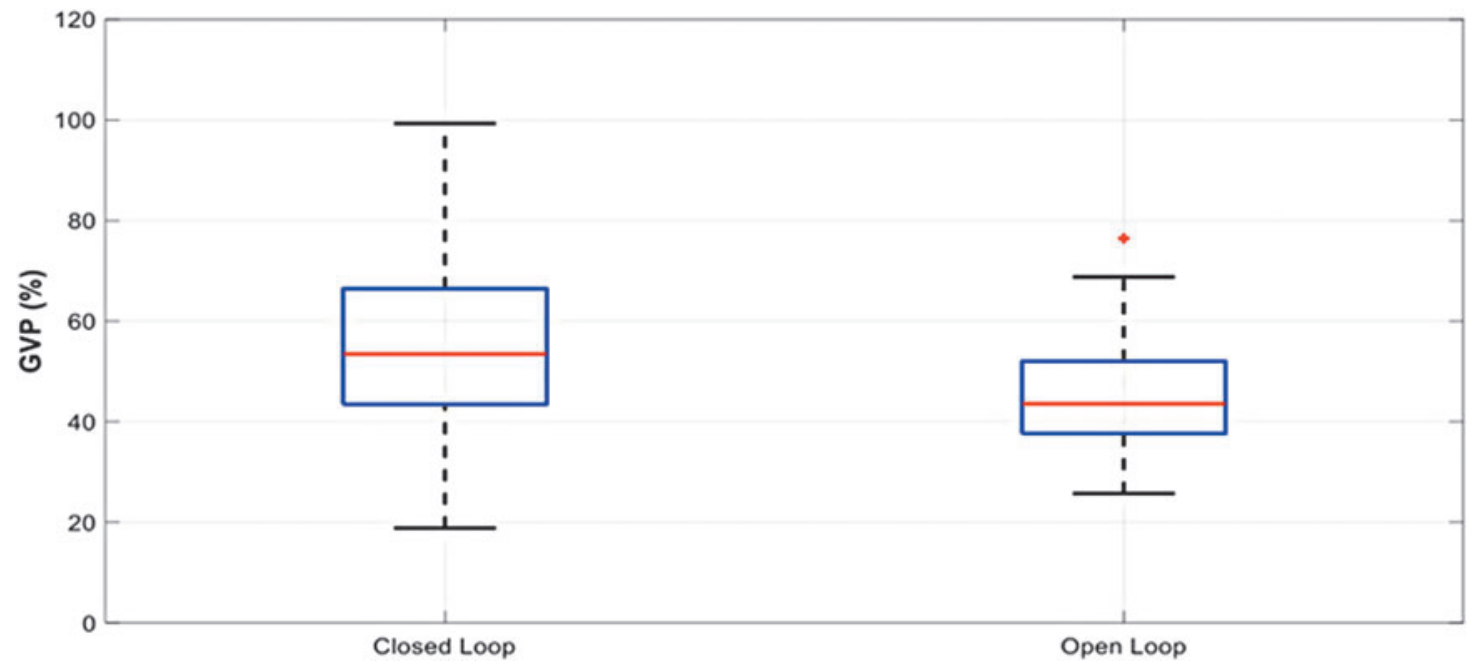

FIG. 6. Box-whisker plot showing higher mean GVP and broader distribution of GVP for the closed-loop arm than the open-loop arm of the bionic pancreas study reported by Russell et al. ${ }^{7}$ 
Table 2. Comparison of OUR Metrics for Glycemic VARIABILITY FOR SUBJECTS ON OPEN LOOP and Closed Loop (Bionic Pancreas)

\begin{tabular}{lcc}
\hline $\begin{array}{l}\text { Glycemic } \\
\text { variability metric }\end{array}$ & Open loop & $\begin{array}{c}\text { Closed loop } \\
\text { (bionic pancreas) }\end{array}$ \\
\hline MAGE & $173.7( \pm 39.2)$ & $145.7( \pm 34.2)$ \\
SD & $66.27( \pm 12.5)$ & $56.08( \pm 12.2)$ \\
CV & $0.420( \pm 0.06)$ & $0.392( \pm 0.07)$ \\
GVP & $46 \%( \pm 11 \%)$ & $55 \%( \pm 17 \%)$ \\
\hline
\end{tabular}

MAGE, SD, and CV with their dependence or weighting on the amplitude of oscillations show a reduction in glycemic variability from open to closed loop.

$\mathrm{CV}$, coefficient of variation; MAGE, Mean Amplitude of Glycemic Excursions; SD, standard deviation.

glucose and glycemic variability. ${ }^{11}$ Reduction in the mean glucose without concomitant reduction in the glycemic variability may put patients at increased risk for severe hypoglycemic events. Patients who experience reduced glycemic variability as a result of using a CGM device may be more likely to continue to use the technology, whereas patients who still struggle with high levels of glycemic variability may be at risk for discontinuing use of the technology.

In a longer study with the second generation Dexcom CGM system, the Dexcom Seven, Rodbard et al. found a reduction in glycemic variability associated with 3 weeks of use of a 7-day continuous glucose monitoring system. ${ }^{12}$ They speculated that the availability of real-time continuous glucose monitoring data enabled patients to "avoid excessively long or severe excursions into the hyper- or hypoglycemic regions" thus effectively reducing the overall glycemic variability. The ability to easily quantify glycemic variability in patients on CGM devices may provide healthcare providers with an additional tool for guiding patients in the optimum use of this technology.

Emerging commercial artificial pancreas systems are also likely to result in decreases in both mean glucose and glycemic variability. As noted above, Russell et al. reported a decrease in both mean glucose and glycemic variability in their study of the bionic pancreas. However, the metric used in their analysis of glycemic variability, the SD, may be underestimating the degree of glycemic variability actually present in the data. Using the GVP metric which captures both the amplitude and frequency of oscillations, subjects in the closed-loop arm of that study were found to have comparable GVPs as subjects with type 1 diabetes in open-loop CGM clinical studies.

Since different metrics of glycemic variability are based on measuring different components of the time-dependent glucose profile, it is also possible that different glycemic variability metrics are advantageous in different situations. For example, SD or CV may be a better metric when assessing the magnitude of postprandial glucose excursions, whereas GVP, which takes into account both frequency and amplitude, may be a better marker for assessing the long-term effects of reactive oxygen species and inflammatory activation on patients. Further research is required to explore these possibilities.

\section{Conclusions}

Historically, mean glucose has been associated with A1c and thus implicitly with long-term clinical outcomes. Indeed, there are widely used formulae for the relationship between A1c and the estimated average or mean glucose. ${ }^{13}$ Nonetheless, glycemic variability is now widely recognized as an important element of glycemic control in addition to mean glucose. Earlier, widely accepted metrics for glycemic variability were based on measures that emphasized the amplitude of glycemic fluctuations. This was appropriate when the principal measurement methodology was intermittent capillary fingersticks. However, given the advent of continuous glucose monitoring and the increasing widespread adoption of the technology, it may be useful clinically to consider other metrics for glycemic variability that take full advantage of the more granular temporal resolution available with CGM technology and include both the frequency and amplitude of glycemic fluctuations.

The new metric proposed in this study and in the companion article ${ }^{1}$ may provide clinicians with the ability to rapidly identify patients who have persistent high levels of glycemic variability and who would benefit from additional support from healthcare providers in the optimum use of CGM technology. In addition, the new metric proposed in this study may be useful for assessing the different levels of performance in different artificial pancreas systems soon to be available to patients. In a previous article, Hirsch et al. expanded the use of the GVP by combining it with the mean glucose, the percent time in range $(70-180 \mathrm{mg} / \mathrm{dL})$, and a hypoglycemic penalty function to provide a single composite metric to capture multiple dimensions of glycemic control based solely on data from continuous glucose monitoring systems. ${ }^{14}$

\section{Author Disclosure Statement}

A.G. and A.K.B are employees of Dexcom. B.A.B. has received research support from and has consulted for Dexcom, Medtronic, Tandem and Bigfoot Medical. I.B.H. is a consultant for Abbott Diabetes Care, Roche, Intarcia, and Valeritas. T.A.P. is the cofounder and chief scientist of ModeAGC LLC and has consulted for Dexcom, Insulet and Tandem.

\section{References}

1. Peyser T, Balo A, Buckingham B, et al.: Glycemic variability percentage (GVP): a novel metric for assessing glycemic variability from continuous glucose monitor data. Diabetes Technol Ther 2017; doi: 10.1089/dia.2017.0187.

2. Marling CR, Shubrook JH, Vernier SJ, et al.: Characterizing blood glucose variability using new metrics with continuous glucose monitoring data. J Diabetes Sci Technol 2011;5:871-878.

3. Hermanides J, Vriesendorp TM, Bosman RJ, et al.: Glucose variability is associated with intensive care unit mortality. Crit Care Med 2010;38:838-842.

4. DeVries JH: Glucose variability: where it is important and how to measure it. Diabetes 2013;62:1405-1408.

5. Peyser T, Dassau E, Breton M, Skyler JS: The artificial pancreas: current status and future prospects in the management of diabetes. Ann N Y Acad Sci 2014;1311:102-123.

6. Garg S, Zisser H, Schwartz S, et al.: Improvement in glycemic excursions with a transcutaneous, real-time continuous glucose sensor: a randomized controlled trial. Diabetes Care 2006;29:44-50. 
7. Russell SJ, El-Khatib FH, Sinha M, et al.: Outpatient glycemic control with a bionic pancreas in type 1 diabetes. $\mathrm{N}$ Engl J Med 2014;371:313-325.

8. Whitelaw BC, Choudhary P, Hopkins D: Evaluating rate of change as an index of glycemic variability, using continuous glucose monitoring data. Diabetes Technol Ther 2011;13:631-636.

9. Kovatchev BP, Clarke WL, Breton M, et al.: Quantifying temporal glucose variability in diabetes via continuous glucose monitoring: mathematical methods and clinical application. Diabetes Technol Ther 2005;7:849-862.

10. El-Laboudi AH, Godsland IF, Johnston DG, Oliver NS: Measures of glycemic variability in type 1 diabetes and the effect of real-time continuous glucose monitoring. Diabetes Technol Ther 2016;18:806-812.

11. Kovatchev B, Cobelli C: Glucose variability: timing, risk analysis, and relationship to hypoglycemia in diabetes. Diabetes Care 2016;39:502-510.

12. Rodbard D, Bailey T, Jovanovic L, et al.: Improved quality of glycemic control and reduced glycemic variability with use of continuous glucose monitoring. Diabetes Technol Ther 2009;11:717-723.

13. Nathan DM, Kuenen J, Borg R, et al.: Translating the A1C assay into estimated average glucose values. Diabetes Care 2008;31:1-6.

14. Hirsch IB, Balo AB, Sayer K, et al.: A simple composite metric for the assessment of glycemic status from continuous glucose monitoring data: implications for clinical practice and the artificial pancreas. Diabetes Technol Ther 2017;19(Suppl. 3):S38-S48.

Address correspondence to:

Arturo Garcia, BS Dexcom, Inc. 6340 Sequence Drive San Diego, CA 92121

E-mail: agarcia@dexcom.com 